\title{
Lubricant Flow in an Elastohydrodynamic Contact Using Fluorescence
}

\author{
T. Reddyhoff ${ }^{1}$, J. W. Choo $^{2}$, H. A. Spikes ${ }^{1}$, R. P. Glovnea ${ }^{3}$. \\ 1. Department of Mechanical Engineering, \\ Imperial College, London, SW7 2AZ, UK. \\ 2. Vestas Technology R\&D, Singapore Pte Ltd \\ 3. School of Engineering \& Design \\ Brunel University, Uxbridge, UB8 3PH, UK.
}

\section{Abstract}

It is well documented that parameters such as film thickness and temperature in EHL contacts can be measured experimentally using a range of techniques include optical interferometry, ultrasonics, capacitance and infrared emission. Considerably less is known, however, about the flow of lubricant through such contacts. Information about lubricant flow would greatly benefit the prediction of friction in machine components.

This paper describes initial steps to develop fluorescence as a means of observing lubricant flow.

An EHL contact was produced between a steel ball and a glass disc and viewed using a fluorescence microscope. The entrained lubricant was dyed using a fluorescent species, so that when illuminated with laser light, a fluorescence intensity map could be viewed. When the contact was fully flooded with dyed lubricant, the fluorescence intensity within the contact correlated well with optical interferometric film thickness measurements under the same conditions. This suggests useful possibilities for mapping film thickness in contacts where conventional optical methods are impractical, such as between rough surfaces and within soft contacts.

In order to observe how lubricant flows in an EHL contact, fluorescer-containing lubricant was placed on the out-of-contact track. The boundary between fluorescent and non-fluorescent lubricant was then entrained into the contact and the passage of the boundary through the contact was monitored.

\section{Key words}

EHD, film thickness, fluorescence, lubricants.

\section{Introduction}

\subsection{Lubricant Flow}

The prediction of friction in EHL contacts is of great practical importance, since it is this friction that determines the power loss in the contact, and therefore the efficiency 
of many machine components. Furthermore, in certain components where operation relies on controlled shear of the lubricant, such as variable transmission applications, the accurate prediction of friction is of critical importance [1]. The latter is, however, not straightforward, since, unlike film thickness which is determined in the inlet; traction arises from the central region of the EHL contact. Here, due to the extreme conditions of high strain rates (up to $10^{8} \mathrm{~s}^{-1}$ ) and pressures (up to $3 \mathrm{GPa}$ ), the lubricant behaves in a highly non-Newtonian fashion. Much effort has been expended in developing realistic constitutive relationships between the stress and the strain rate of the lubricant in EHL conditions [2], based mainly on disc machine measurements and high pressure viscometry. There is however still considerable dispute regarding the choice and validity of such models and in particular between models derived from disc machine versus viscometer measurements [3]. This problem is due in part to the lack of understanding of how the lubricant flows through the contact.

It is a challenge to experimentally map lubricant flow through an EHL contact. The lubricant film is extremely thin $(<1 \mu \mathrm{m})$ so it is generally not practicable to observe it from the side. The use of particles to reveal flow trajectories is difficult since, because the film is thin the particles must be very small and there is also the possibility of particles segregation in the flow field. Finally the duration of flow of lubricant through the contact is very short $(\sim 1 \mathrm{~ms})$. One study has been carried out by Bair and co-workers, where a compressed layer of lubricant $\sim 150 \mu \mathrm{m}$ in thickness into which small particles were dispersed was subject to shear. Because the lubricant layer was very thick it could be viewed from the side using a microscope, enabling velocity profiles across the film to be obtained. At low shear rates, mechanicallyinduced slip bands were observed at an angle of about $20^{\circ}$ to the surfaces [4], similar to those experienced by bulk yield of solid polymers [5]. These shear bands increased in number with increasing shear rate. At higher pressures, shear deformation was localised at the centre of the film, where a thin hot layer of liquid was sandwiched between two cooler layers [6]. Unfortunately, in these experiments, the film thickness was extremely thick $(\sim 150 \mu \mathrm{m})$, much thicker than those found in EHL contacts and it is difficult to see how a similar experimental approach could be applied to realistic EHL film thicknesses.

One possible mode of fluid flow is slip, where the fluid at the wall of the contact has a different velocity to that of the surface bounding it. The occurrence of such slip has been demonstrated between smooth surfaces under hydrodynamic lubrication [7, 8]. Through film thickness measurement, Kaneta and co-workers have inferred that slip occurs in EHL [9]. However this is interpretation is indirect and has been contested [4].

The aim of this work is to image lubricant flow in EHL contacts. An approach using fluorescence is employed.

\subsection{Fluorescence}

A fluorescent substance can absorb light at one wavelength (e.g. blue), then subsequently emit light of another wavelength (e.g. green). As described by Haugland [10], fluorescence occurs in three stages:

1) Excitation. A photon supplied by an external source is absorbed by a fluorophore creating an excited electronic singlet state (a single electron is promoted). 
2) Excited-state lifetime. The molecule exists in such a state for 1-10 ns, during this time it may undergo relaxation to leave it in a state from which it may emit fluorescence. There are other means by which molecules return to their ground state (e.g. coalitional quenching, fluorescence energy transfer and intersystem crossing). The quantum yield, which is the ratio of the photons absorbed to the photons of fluorescence emitted, gives a measure of the relative levels of these processes.

3) Fluorescence Emission. A photon of energy is emitted as the fluorophore returns to its ground state. Due to energy dissipation during the excited-state lifetime, the energy of this photon is lower, and therefore of longer wavelength, than the excitation photon. This difference in wavelength is known as Stokes shift and is important in fluorescence applications since it allows separation of the measured emission from excitation.

The wavelengths and intensities at which fluorophores absorb and emit are determined by their electronic structure. A clear explanation of the link between chemical structure and absorption and emission is given by Sharma [11]. Many lubricants contain molecular features such as double bonds which allow for absorption in the ultra-violet and re-emission in the visible [12]. However for reasons of increased emission intensity and to be able to select the desired excitation and emission wavelengths, commercially available fluorophores, or dyes are used in this work. Typically, the absorption and emission spectra for a commercial dye take the form shown in figure 3. Such dyes can be dissolved in liquids and result in solutions where fluorescence is visible even at very low concentrations. An alternatively approach used in biological applications is to tag a fluorescent probe to a specific molecule, in order to monitor its movement.

Fluorescence microscopy is used extensively in biological and medical applications, which will not be discussed here; a thorough review of the most commonly used fluorescent protein is given by Tsien [13]. A fundamental difference in approach between biological applications of fluorescence and those for lubricant flow proposed in this paper is that the latter involves events of considerably shorter duration; $\sim 1 \mathrm{~ms}$ rather than the diffusion of species in cells, which occur over a longer time scale.

\subsection{Fluorescence in Tribology}

As a tool to measure film thickness, fluorescence has several advantages over other more established techniques. A feature of this approach is that, whereas interferometry measures the separating gap between surfaces, fluorescence measures the actual amount of lubricant present between two surfaces [14]. This means that fluorescence has the potential to give information of lubricant behaviour through the thickness of the lubricant film - an ability lacking in optical interferometry. Secondly, fluorescence measurements require no reflective coating of specimens, and so can potentially allow measurement of film thickness between rough surfaces. Thirdly, modern visible light photon detection equipment is extremely sensitive, so that very low levels of fluorescence emission down to as little as that emitted from one molecule can be measured. Despite these strengths, fluorescence has been used only sparingly to study lubricant behaviour.

Fluorescence was first applied to tribology in the 1970s, where Ford and co-workers studied free lubricant films on roller and raceway surfaces in rolling element bearings 
[12]. Their method relied on natural fluorescence in the oil, which emitted in the visible range when excited by a UV mercury lamp. The technique was subsequently improved by replacing the mercury lamp with a He-Cd laser, which had associated benefits of simplified optics and increased working distance [15]. Films down to 1 $\mu \mathrm{m}$ thickness were measured.

Since Ford's work, there have been a number of other studies based on fluorescence. Most of these have used the technique of laser-induced fluorescence (LIF), where a fluorescent species is excited by a laser at one frequency and the intensity of emission at a different frequency is detected to indicate the fluoresce concentration and thus the lubricant film thickness. Shaw et al. [16][17] and Richardson and Borman [18] applied LIF to monitor film thickness between piston and liner. The emitted light was transmitted from the contact through the cylinder liner by means of a fibre optic cable. One strength of this approach is that there is effectively no upper bound on measured film thickness, it is robust regarding temperature variation and it can measure both the film thickness in the piston/liner contact and also the lubricant on the out-of-contact cylinder surface. Tanimoto et al have used laser-induced fluorescence to map the migration of free surface films from the wear scars on hard disks [19].

Fluorescence has been used extensively to measure film thickness in sealing applications, because it is either important to know the volume of lubricant present (rather than the separation of surfaces), or large film thickness mean optical interferometry is not possible [20, 21, 22, 23, 24]. A notable example of this is research by Poll and co-workers [20], where an actual rotary seal was run against a glass shaft. Here film thicknesses as low as $0.35 \mu \mathrm{m}$ were measured. Work carried out at Luleå University measuring film thickness in hydraulic cylinder seals, is summarized in reference [22], which demonstrates the ability of laser induced fluorescence to study soft contacts, whose optical properties preclude the use of optical interferometry. An interesting technique that uses two fluorescent dyes has been developed for rotary seals by Hidrovo and co-workers [24]. One dye absorbs incident laser light, and subsequently fluoresces providing the excitation for a second dye. In this way film thickness is obtained independently of background noise. This technique is currently limited in its application since it requires a thick fluorescent film to operate.

There has been very little application of fluorescence to concentrated elastohydrodynamically lubricated (EHL) contacts, probably because optical interferometry already provides a powerful means of analysing such small contact areas. To the authors knowledge, the only example of fluorescence applied to a concentrated contact has been work by Sugimura et al [25, 26]. They reported problems of interference and sensitivity. The former they were able to model but sensitivity issues were more problematic. Further details of the problem of interfering of light emitted by the fluorescently dyed lubricant can be found in the following reference [27]. However since this work, photon detection equipment, as well as fluorescent dye technology has advanced significantly.

An extremely insightful application of fluorescence to study lubricant behaviour has been carried out by Pit et al. in order to study liquid slip at solid surfaces [7]. Pit used a process known as fluorescence recovery after photo-bleaching (FRAP). FRAP was first demonstrated by Axlerod [28], and is achieved as follows. A relatively unstable fluorescent dye is added to the fluid. Initially a tightly-focussed, high power laser is used to illuminate the fluid; this has the effect of destroying the dye molecules inside 
the small illuminated region. Then the sample is illuminated using a much lower power laser, causing the non-bleached molecules present to fluoresce. Subsequent diffusion and flow of the surrounding fluorescent fluid sample into the bleached area can then be monitoring. Pit used this rate of replenishment to prove that lubricant slip at the wall occurred between two sliding flat plates. The ultimate aim of the current research is to apply FRAP to an EHL contact in order to investigate shearing and slip. In this paper however, only a simpler method of dye introduction, which mimics a FRAP experiment, is presented.

\section{Experimental Technique}

\subsection{Fluorescence Apparatus}

A lubricated contact is produced using a conventional optical interferometric, ball on disc test rig (PCS Instruments Ltd, Acton, UK), where a steel ball is loaded against a glass disc, as shown in figure 1. Both ball and disc can be rotated independently to give a range of slide-roll-ratios, however only pure rolling conditions were used in this work. The lubricant is held in a temperature-controlled bath $\left(+/-0.5^{\circ} \mathrm{C}\right)$ and the ball is half-immersed in lubricant to ensure fully-flooded conditions.

In the current work, the contact was located beneath a fluorescence microscope (Axovaria manufactured by Zeiss, Jena, Germany), with a 20x Olympus Neofluor objective. Excitation was provided using a solid-state, diode-pumped pulsed laser, which produced a beam of wavelength $532 \mathrm{~nm}$ (Laser2000 Ltd, Northants, UK). It has a repetition rate of 0.2 to $20 \mathrm{kHz}$ and average maximum power output of $40 \mathrm{~mW}$ at $3 \mathrm{kHz}$, which is sufficiently low to ensure no bleaching of the dyed lubricant over the duration of the test.

A high speed camera with a built-in image intensifier (Focusscope SV200-i, manufactured by Photron Ltd, West Wycombe, UK) was mounted above the beam splitter so that it received the emitter fluorescence from the contact. The intensifier was required since a relatively small amount of light was emitted from the contact during the required $4 \mu \mathrm{s}$ exposure. Each light pulse emitted from the laser was synchronised to fall within the exposure of the high speed camera, so that no erroneous fluctuations in measured intensity (beating) occurred.

As described in section 3.2, the fluorescent light emitted from the contact is of longer wavelength than the excitation of the laser. This allows the separation of the two light beams by a dichroic beam splitter located between the objective and camera as shown in figure 1. The characteristics of the beam splitter as well as the laser and dye wavelengths are shown in figure 3.

Stop 1 was opened fully for all tests. Stop 2 was opened sufficient to illuminate/view the circular contact area. Other optical components Lens 1, Filter 1 (used to allow only $532 \mathrm{~nm}$ light through), the beam expander and tilting mirrors used for laser beam alignment were mounted onto a common rail with the laser source. 


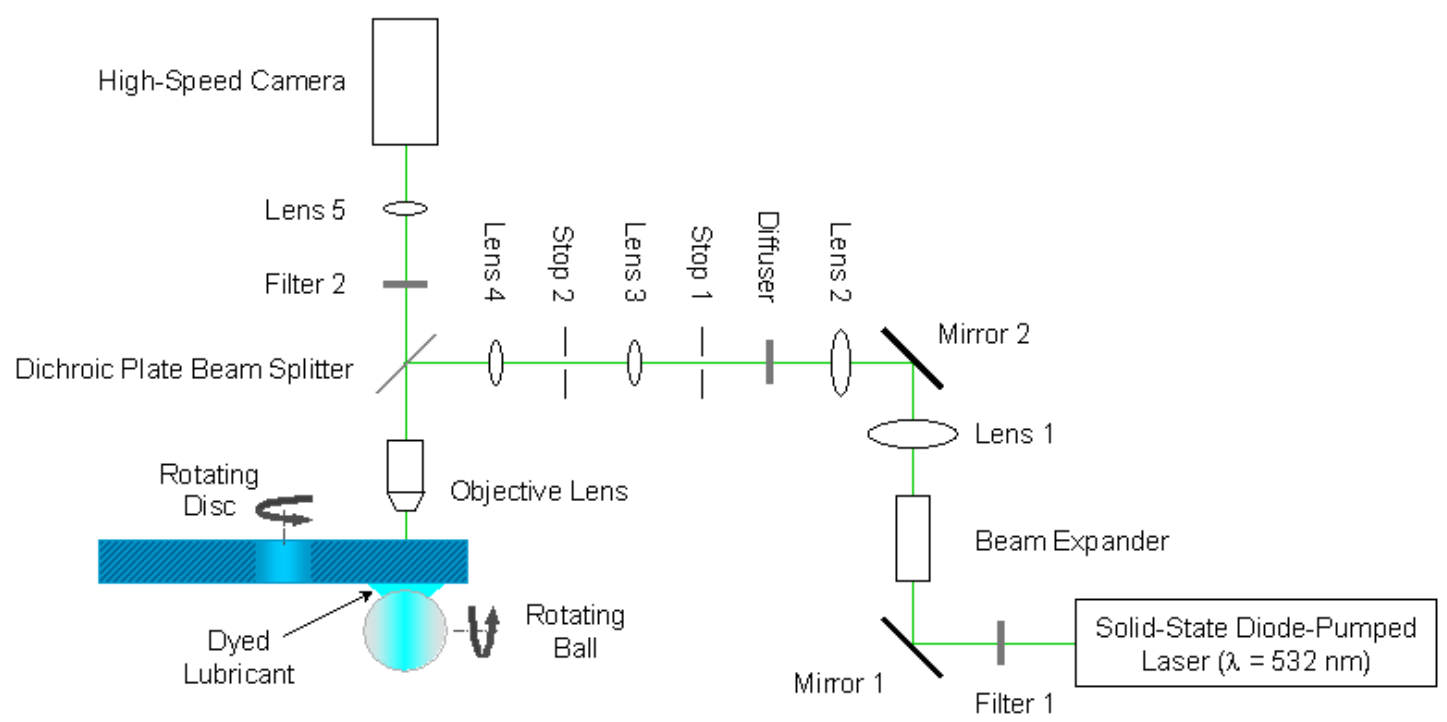

Figure 1. Schematic of optical set-up and EHD contact.

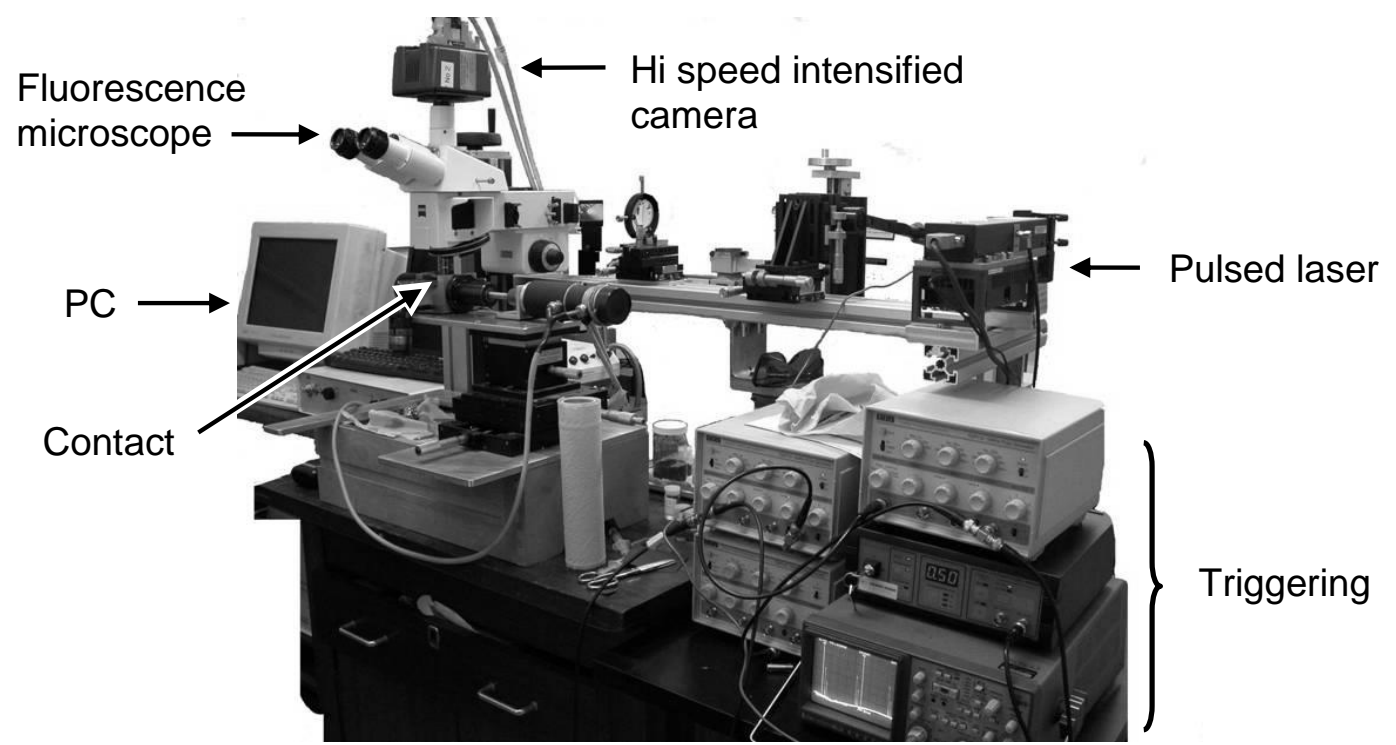

Figure 2. Photograph of apparatus.

\subsection{Lubricant-Dye Combinations}

Glycerol was used as the lubricant for the majority of testing, since its polar nature allows it to dissolve a range of commercially-available dyes. For this work, the dye Eosin was dissolved at a concentration of $0.04 \%$ by mass. Eosin was chosen since its absorption peak coincided with the excitation wavelength of the available laser (532 $\mathrm{nm}$ ) and its quantum yield was sufficiently high to result in bright, clearly-defined images of the contact. The absorption and emission spectra for Eosin are shown in figure 3, alongside the characteristics of the dichroic beam splitter used to separate excitation and emitted light.

Due to the difficulty in identifying reasonable cost, hydrocarbon-soluble dyes which fluoresced at the wavelength of the laser excitation, the option of using the natural fluorescence of un-dyed lubricant was considered (as used by Smart et al [12]). This approach was not implemented however since the high intensity afforded by a 
commercially available dye was required in order to capture images of the thin lubricant films being studied. An additional advantage of using a dye is that the intensity of the emitted fluorescence can be precisely controlled by varying the concentration of dye used.

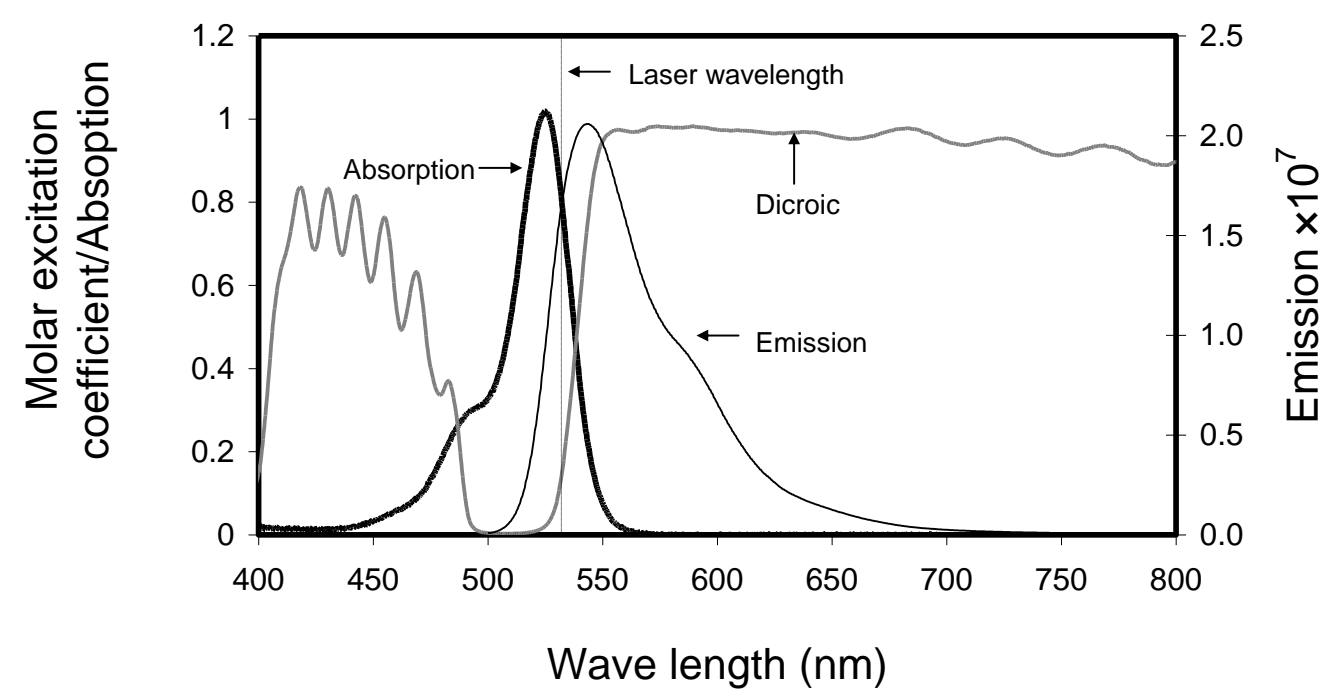

Figure 3. Absorption and emission spectra for Eosin dye [29]. Filter characteristics and laser wavelength are also shown.

\subsection{Dye Entrainment Method}

In order to observe how lubricant flows though a contact, the following procedure was implemented. Initially, a clean disc was loaded against steel ball, and the lubricant bath filled with pure (un-dyed) glycerol; while a single drop of fluoresce-containing glycerol was carefully placed on the lower surface of the otherwise clean disc, upstream of the contact. Then as the disc was subsequently rotated, the dyed portion of lubricant moves towards the contact before reaching the meniscus, and being entrained. Images of the entrained fluorescence were then recorded to show the path of the lubricant through the contact. This was possible since differences in physical properties between the fluorescer-free and fluorescer-containing lubricant are negligible at the fluorescer concentration used.

A source of error in this approach is the diffusion of dye due to random molecular motion. It is accepted that diffusion occurs in the period of time between the dyed lubricant reaching the meniscus and inlet of the contact; what is critical however is the extent that random diffusion occurs as the dye passes through the contact, since this effect will mask the observed mixing due to fluid flow. The extent of random diffusion can be calculated by solving Ficks' second law, giving the variation in concentration with time and distance from the point of contact between dyed and undyed lubricant proportions (at the meniscus).

For the entrainment speed of $0.075 \mathrm{~ms}^{-1}$ that was used, the time taken for the fluid to flow through the contact is of the order of $5 \mathrm{~ms}$, giving change in dye concentration due to diffusion during this time is found to be less than $1 \%$. This is because the time taken for the dye to pass through the contact is very small compared to the time for the dye to pass through the meniscus. The solution of Ficks' second law used here, 
has been omitted for brevity however, it can be found in most textbooks on diffusion, for instance [30] (pp. 28-31).

The diffusion coefficient used in the above calculation is that for glycerol, which at atmospheric pressure has a value of $0.06 \mathrm{~m}^{2} \mathrm{~s}$ [31]. However it has been shown [31], that diffusion coefficients for glycerol are nearly inversely proportional to liquid viscosity. Therefore diffusion of the dye will be further reduced due to the elevated viscosities present within the contact.

\section{Results and Discssion}

Figure 4 shows a fluorescence intensity map obtained from a contact fully-flooded with Eosin-doped glycerol in steady state conditions. If film thickness measurement was the objective of these tests, this intensity map could be converted to a map of film thickness by applying a calibration. Such a calibration would be achieved by plotting the known film thickness versus fluorescent intensity from a fluorescence image of the ball loaded statically against the disc as was done by Sugimura [26]. From figure 4 , the typical horseshoe shape is clearly visible. The dark region at the outlet of the contact is due to cavitation; around which streamers can be seen. Since the presence of lubricant in this region is reduced, it appears darker. This highlights a further possible area of study using fluorescence, i.e. study of the separation of lubricant between ball and disc once it leaves the rear of the contact.

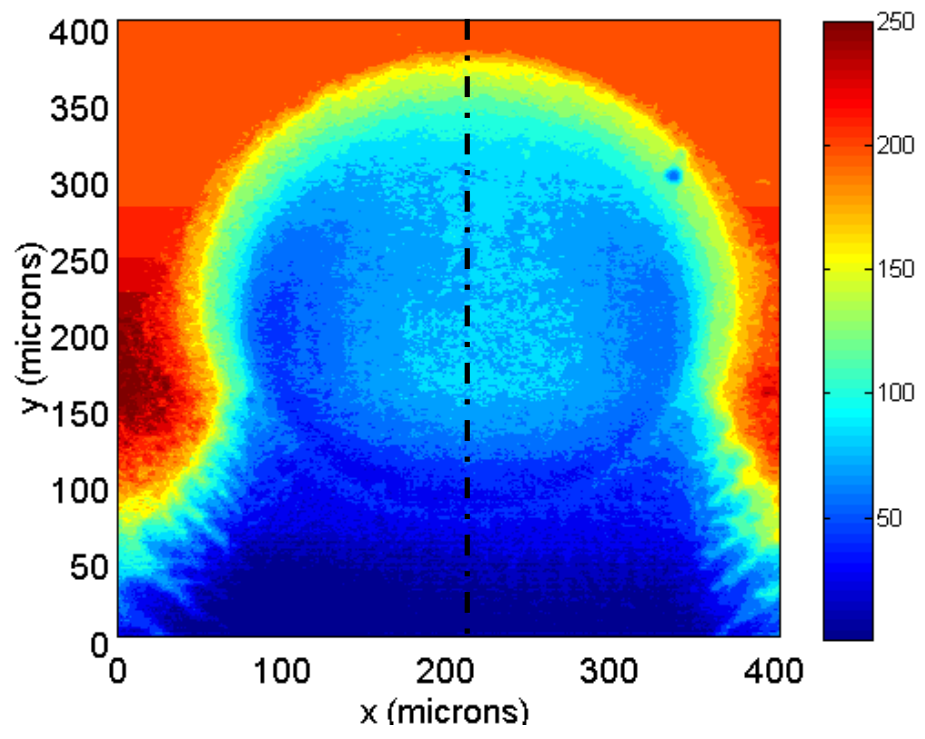

Figure 4. Fluorescence maps of pure rolling contacts subject to $0.075 \mathrm{~m} / \mathrm{s}$ entrainment speed. The contact is lubricated with Eosin, $0.05 \%$ wt. in Glycerol. The temperature of lubricant at inlet is $23^{\circ} \mathrm{C}$ and load is $20 \mathrm{~N}$. The inlet is located at top of the image.

Figure 5 shows the profile of intensity taken along the dashed line in figure 4 , where the typical film shape and constriction can be seen at the outlet. Also plotted in figure 5 is the optical interference measurement of film thickness for the contact lubricated under the same conditions. Although the fluorescent intensity is not calibrated to film thickness, there is clearly a good match between the two measurements. It should be noted that fluorescent intensity gives a measure of the amount of lubricant present, 
rather than the gap thickness. Therefore within the cavitated region fluorescence and optically measured thickness cannot be compared, and have not been plotted in figure 5.

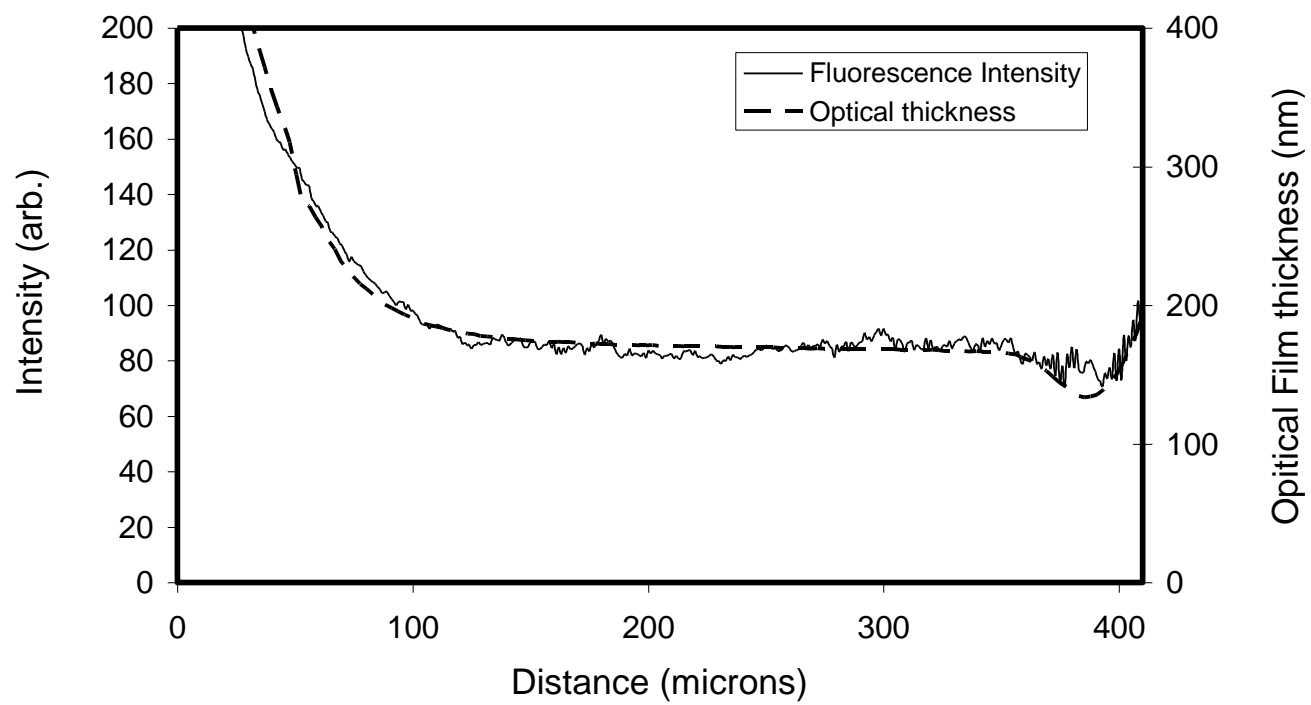

Figure 5 profile down the centre of the contact. Conditions are the same as those for figure 4 . The inlet is at the left.

Figure 6 shows the fluorescent maps obtained when the fluorescent dye entrainment technique described in section 4.3 was carried out. The lubricant progression through the contact is evident, from time $=0$, when no dye is present in the contact, to $5.5 \mathrm{~ms}$ later when the contact is flooded with dye. The intensity in the plots depends on both the film thickness and the presence of dye within the contact. 

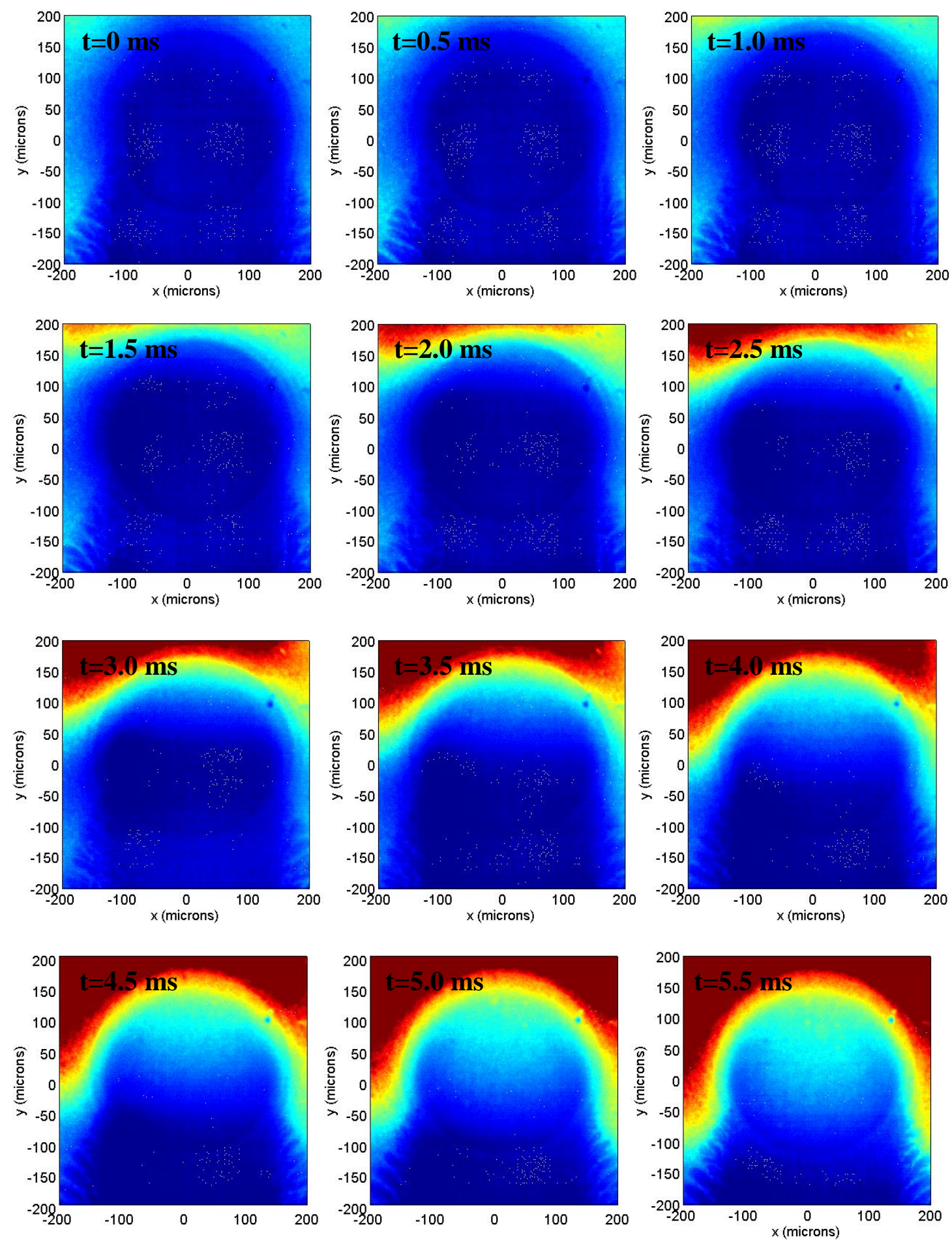

Figure 6. Progression of dye through contact, under pure rolling, subject to $0.075 \mathrm{~m} / \mathrm{s}$ entrainment speed. The temperature of oil at inlet is $23^{\circ} \mathrm{C}$ and load is $20 \mathrm{~N}$. Images taken at $0.5 \mathrm{~ms}$ intervals. The inlet is located at the top of each image

Figure 7 shows fluorescence intensity profiles across the centre of the contact (from inlet on the left to outlet) for each of the fluorescent images shown in figure 6 . The uppermost, bold line shows the intensity when the contact is flooded with dyed lubricant at $5.5 \mathrm{~ms}$. The other lines are angled due to the diffusion of the dye prior to entering to contact, which blurs the dyed/undyed lubricant boundary. If there was a perfect boundary between dyed and un-dyed lubricant, vertical lines would be expected within the contact region. (This is a shortcoming of the method, which 
would be avoided if a technique (such as FRAP) were used to bleach a well-defined region within the contact.) However Figure 7 still provides valuable information. Firstly the intensity lines within the contact are roughly parallel. This shows that no mixing of lubricant is occurring within the contact (in the direction of sliding). This is expected for pure rolling in the very short time available.

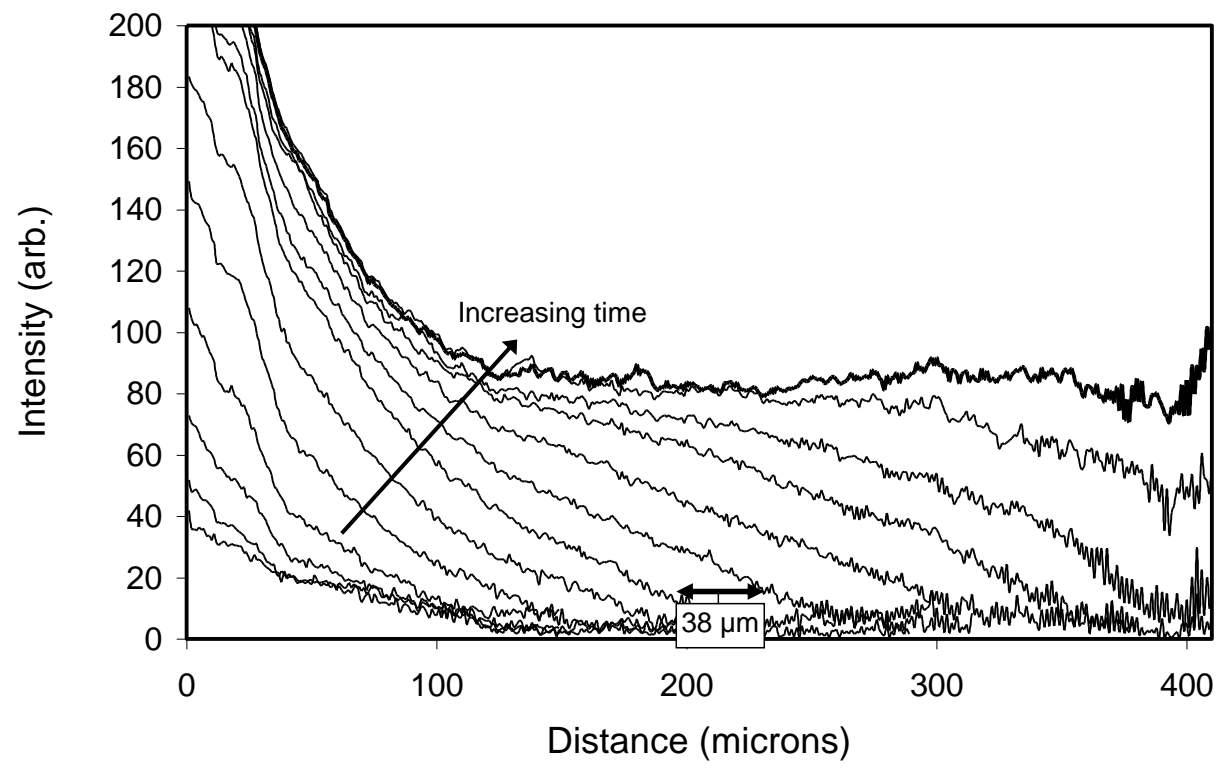

Figure 7. Progression of dye through contact, profile plots. Inlet at left.

A simple validation can be applied to the data shown in figure 7 . The distance the lubricant should move between each measurement line is given by (entrainment speed)/(frame rate of camera). These values are $0.075 \mathrm{~m} / \mathrm{s}$ and 2000 frames per second respectively, which give a distance of $38 \mu \mathrm{m}$. This distance is marked on the $\mathrm{x}$ axis of figure 7 and roughly agrees with the data plotted.

The intensity within each map of figure 6 is due to a combination of four factors.

(i) Film thickness, this is clearly shown by the steady state image after $5.5 \mathrm{~ms}$ where the contact is fully flooded with dye.

(ii) Flow of the dyed lubricant, since this is responsible for the variation between images in figure 6 .

(iii) The degree to which the dyed and un-dyed sections of lubricant are mixed prior to entering the contact; this is demonstrated by the lines in figure 7 not being vertical.

(iv) Distribution of dye through the thickness of the film.

It is of interest to isolate the lubricant flow (ii), from the other effects. In order to do this, each intensity map plotted in figure 6 (i.e. each array of intensity values) was first normalised by dividing every intensity by the corresponding intensity in the final fully flooded map (the image taken at $5.5 \mathrm{~ms}$ ). This was done to remove the effect of film thickness, and is possible since the observed intensity is assumed to be proportional to the thickness of the film. From each of these normalised intensity maps, a contour at a constant level of 0.5 was extracted. Within the contact, each contour is perpendicular to the flow direction, and represents how far through the contact the dye has travelled. These contours are plotted in Figure 8. 


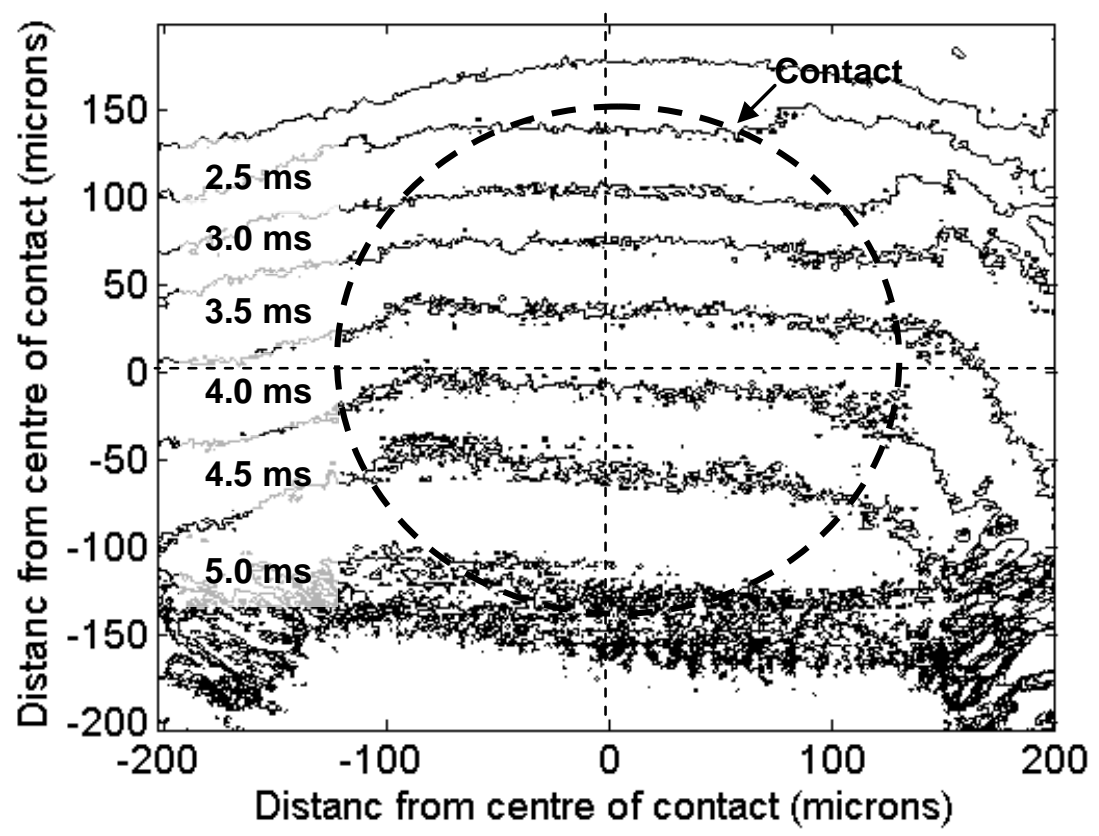

Figure 8. Contours showing progression of dye through the contact. Inlet at top.

It can be seen that the contours across the contact shown in figure 8 are approximately level and parallel over most of the contact, but wedged forward at the sides. This indicates that the mean flow velocity across the central zone of the contact is constant. This is as expected for a rolling EHL contact, where there is very little side leakage and the film thickness is near constant across the film.

From figure 8 it appears that within the contact, the contours become further apart during passage through the contact. This implies that the mean, measured fluid front speed increases across the contact. Figure 9 plots the calculated mean speed based on figure 8. In order to find the lubricant's mean speed as it flows through the contact, the following procedure was applied to the contour data shown in figure 8. A line was fitted to each set of contour data within the central region of the contact $(-75<x$ $<+75 \mu \mathrm{m})$. The average distance between each fitted line was then divided by the period of time between camera exposures to give the mean lubricant speed. The results are shown in figure 9, where each $y$-error bar corresponds to the standard deviation of the noisy contour data about each fitted line used in the speed calculation.

According to this plot, the lubricant speed just before the inlet is close to the entrainment speed. Then as it travels through the contact, it increases from a value $25 \%$ less than the entrainment speed in the inlet region to a value $40 \%$ more than the entrainment speed in the exit region. 


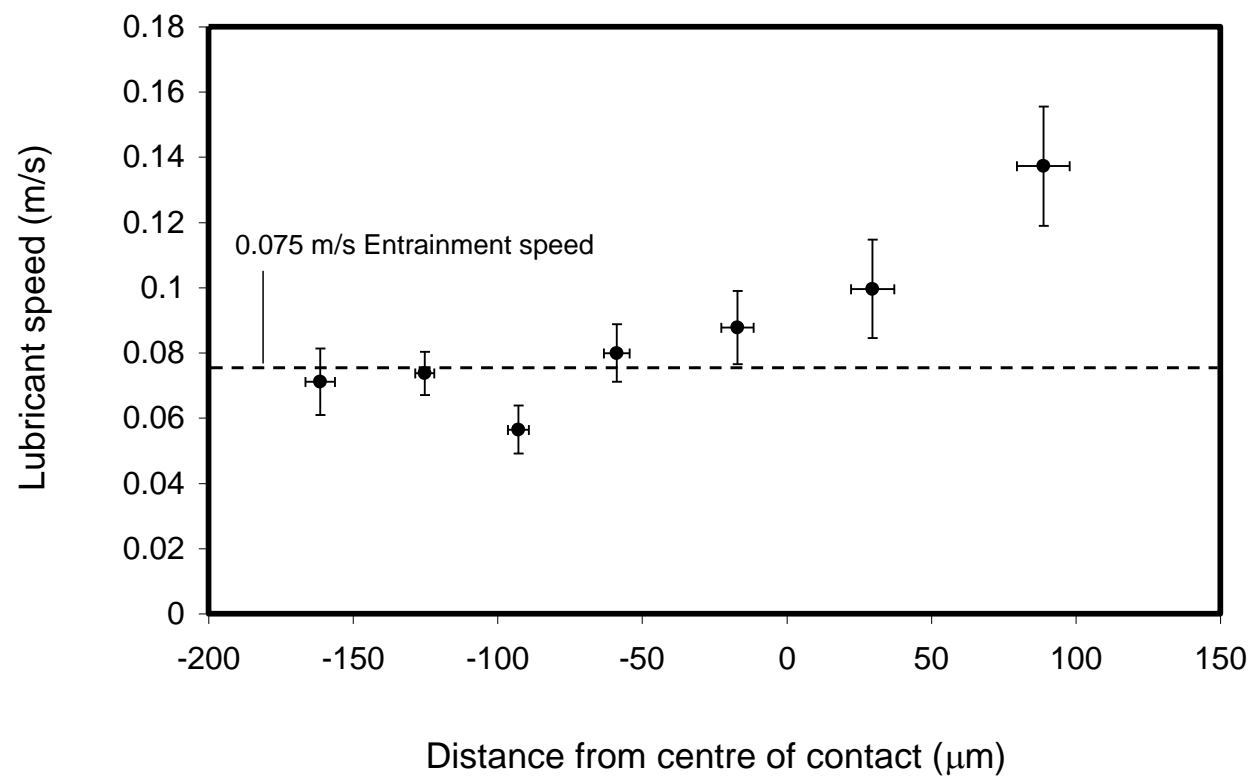

Figure 9. Plot showing the speed of the lubricant as it passes through the contact.

The origins of this quite large effect are not clear. If there is negligible side leakage, then the flow rate in the rolling direction should also be constant. According to Reynolds equation this flow rate along $q_{x}$, and across $q_{y}$ a pure rolling contact is given by;

$q_{x}=U h-\frac{h^{3}}{12 \eta} \frac{d p}{d x}$

and

$q_{y}=\frac{h^{3}}{12 \eta} \frac{d p}{d y}$

where $U$ is the entrainment speed, $\eta$ the dynamic viscosity (at the local pressure) and $h$ the local film thickness. Despite the large values of $\mathrm{d} p / \mathrm{d} x$ in the inlet and exit regions, the extremely large value of $\eta$ should mean that the second term in equation 1 remains very small, so that only small variations in film thickness and thus the first term in equation 1 are enough to balance the flow equation. The average velocity of fluid through the contact (given by $q_{x} / h$ ), is therefore very close to the entrainment speed $U$. This disagrees with the variation in flow velocity shown in figure 9 .

Similarly, the magnitude of $\eta$ in equation 2 means that flow in the y direction is very small. This observation is supported by the contour lines within the contact in figure 8 being generally parallel and horizontal.

As indicated in figure 7, the film thickness in the inlet region of the contact is slightly larger (by about 5\%) than the contact centre. This will produce a slightly reduced 
mean fluid velocity in the inlet, but, in the absence of side leakage, this should scale with the change in film thickness and be much less than the $25 \%$ reduction observed.

The increase in the speed of lubricant through the contact, shown in figure 9 is strikingly similar to that observed in EHL contacts by Glovnea and Spikes [32]. In their work, film thickness profiles were measured using ultra-thin film interferometry during the start up of motion. The development of the profile was then used to chart the speed of motion of the fluid film through the contact, where they note: "In pure rolling conditions, the first film front travels through the conjunction with a speed lower than the entrainment in the first half of the contact and higher in the second half". The magnitude of this speed variation is also similar to that observed in the current work. It should be noted that Glovnea's results are for start up whereas the current work is carried out under steady state conditions. The former is however, the only experimental measurement of fluid velocity available for comparison.

One possibility is compression of the lubricant as it enters the contact, which will reduce volume flow rate and thus velocity. This effect however should also be much less than the observed one. The effect is also too large to be due to microslip.

\section{Conclusions}

Fluorescence microscopy has been applied to study EHL contacts. Intensity maps of the contact obtained correlated well with film thickness measured by optical interferometry, at a central film thickness of $\sim 200 \mathrm{~nm}$. Although the use of fluorescence to measure lubricant film thickness is an established approach, to the authors' knowledge, it has not been shown to work at such small film thicknesses. This demonstrates the aptitude of fluorescence to measure small film thicknesses in concentrated contacts, especially in cases where conventional interferometric technique are problematic such as with non-reflective or rough surfaces.

A dye introduction technique has been developed which is able to reveal the speed of lubricant flow through the contact. In pure rolling conditions this indicates broadly constant flow along the direction perpendicular to entrainment. The technique described is under development, and the results are not completely conclusive, due mainly to mixing of dyed and un-dyed portions of the lubricant before entering the contact. In ongoing work, the microscope set up is being refined to incorporate a high power laser in order to carry out photo-bleaching experiments.

\section{Acknowledgements}

The authors are very grateful to the Mr Adrian Walker and the EPSRC equipment pool for advice, and loan of the high speed camera, without which this work would not have been possible.

\section{References}

[1] Jacod, B., Venner, C. H., Lugt, P. M., “A generalized traction curve for EHL contacts", Trans. ASME, J. Trib., 2001, 123, 2, pp. 248-253. 
[2] Olver, A. V., Spikes, H. A., "Prediction of traction in elastohydrodynamic lubrication", Proc Instn. Mech. Engrs. Part J, 1998, 212, pp. 321-332.

[3] Spikes, H. A., Anghel, V., Glovnea, R., "Measurement of the rheology of lubricant films in elastohydrodynamic contacts', Tribology Letters, 2004, 17(3), pp. 593-605.

[4] Bair, S., Qureshi, F., Winer, W. O., "Observations of shear localization in liquid lubricant under pressure”, ASME J. Trib., 1993, 115, pp. 507-513.

[5] Imai, Y., Brown, N., "Environmental crazing and intrinsic tensile deformation in PMMA”. J . Mater. Sci., 1976, 11, pp. 417-424.

[6] Bair, S., Qureshi, F., Khonsari, M., "Adiabatic shear localization in a liquid lubricant under pressure”, ASME J. Trib., 1994, 116, pp. 705-708.

[7] Pit, R., Hervet, H., L'eger, L., "Friction and slip of a simple liquid at a solid surface", Tribology Letters., 1999, 7, pp. 147-152.

[8] Choo, J.H., H.A. Spikes, M. Ratoi, R. Glovnea, A. Forrest., "Friction reduction in low-load hydrodynamic lubrication with a hydrophobic surface", Tribology international, 2007, 40(2), pp. 154-159.

[9] Kaneta, M., Nishikawa, H., Kameishi, K., "Observation of wall slip in elastohydrodynamic lubrication”, ASME J. Trib. 1990, 112, pp. 447-452.

[10] Haugland, R. P., "Handbook of Fluorescent Probes and Research Chemicals" 1999, 7th edn (Eugene, OR: Molecular Probes)" pp. 1.

[11] Sharma, A., Schulman, S. G., "An introduction to fluorescence spectroscopy", 1999, 190 p., Wiley, New York.

[12] Smart, A. E., Ford R. A. J., "Measurement of thin liquid films by a fluorescence technique", Wear, 1974, 29, pp. 41 - 47.

[13] Tsien, R. Y., “The green fluorescent protein” Annu. Rev. Biochem. 1998. 67, pp. 509-544

[14] Spikes, H. A., "Thin films in elastohydrodynamic lubrication: the contribution of experiment”, 1999, Proc Instn. Mech. Engrs. Part J, 213, pp. 335-352. 
[15] Ford R. A. J., Ford, C. A., "Laser-based fluorescence techniques for measuring thin liquid films", Wear, 1978, 51, pp. 289 - 297.

[16] Shaw, B., T. II., Hoult, D. P, Wong, V. W., "Development of engine lubricant film thickness diagnostics using fiber optics and last fluorescence", SAE 920651.

[17] Shaw, B., T. II., Lux, J. P, Wong, V. W., "Calibration of laser fluorescence measurements of lubricant film thickness in engines", SAE 881587.

[18] Richardson, D. A. and Borman, G. L., "Using fibre optics and laser fluorescence for measuring thin oil films with applications to engines". International Fuels and Lubricants Meeting and Exposition, 1991, Toronto, Publ by SAE.

[19] Tanimoto, K., Rabinowicz, E., "A fluorescence technique for measuring lubricant thickness on hard magnetic disks", Tribology Transactions, 1992, 35(3), pp. 537-543.

[20] Poll, G., Gabelli, A., Binnington, P. G., Qu, J. Dynamic mapping of rotary lip seal lubricant films by fluorescent image processing. In Proceedings of the 13th BHR Group International Conference on Fluid Sealing, Brugge, Belgium, 1992, pp. 55-77.

[21] Toda, A., Nakamura, K., "A Study of the Sealing Mechanism of Radial Lip Seal With Helical Ribs-Measurement of the Lubricant Fluid Behavior Under Sealing Contact" SAE 1999-01-0878.

[22] Jacobson, B., "Rheology and elastohydrodynamic lubrication", Elsevier. Sic New York, 1991.

[23] Ayala, H., M., Hart, D. P., Yeh, O. C., Boyce, M. C., "Wear of elastomeric seals in abrasive slurries", Wear, 1998, 220, pp. 9-21.

[24] Hidrovo Chavez, C. H., Development of a fluorescence based optical diagnostic technique and investigation of particle ingestion and accumulation in the contact region of rotating shaft seals. Doctoral Thesis. Massachusetts Institute of Technology, 1995.

[25] Sugimura, J., Yoshimizu, I., Yamamoto, Y., "Visualisation of lubricating films by fluorescence microscope" (in Japanese). In Proceedings of JAST Tribology Conference, Tokyo, May 1993, pp. 143-146.

[26] Sugimura, J., Yoshimizu, I., Yamamoto, Y., "Visualisation of lubricating films by fluorescence microscope", In Proceedings of JAST Tribology Conference,Tokyo, 1993, pp. 143-146. 
[27] Sugimura, J., Hashimoto, M., Yamamoto, Y., Study of elastohydrodynamic contacts with fluorescence microscope, Proc. 26th Leeds-Lyon Symposium on Tribology, Thinning Films and Tribological Interfaces, D. Dowson et al. eds., Elsevier, 2000, pp.609-617.

[28] Axelrod, D., Koppel, D. E., Schlessinger J., Elson, E., Webb, W., "Mobility measurement by analysis of fluorescence photobleaching recovery kinetics", J. Biophysical, 1976, 16, pp. 1055-1069.

[29] Du, H., Fuh, R. A., Li, J., Corkan, A., Lindsey, J. S., "PhotochemCAD: A computer-aided design and research tool in photochemistry," Photochemistry and Photobiology, 1998, 68, pp. 141-142.

[30] Cussler, E. L., "Diffusion, mass transfer in fluid systems", $2^{\text {nd }}$ Edn, p.580, , Cambridge University Press, Cambridge, 1997.

[31] Evanoff, J. E., Harris, W. E., "Diffusion coefficients and viscosities in glycol and glycerol solutions", 1974, Canadian Journal of Chemistry, 56, pp. 574-577.

[32] Glovnea, R. P., Spikes, H. A., "Elastohydrodynamic film formation at the start up of motion" 2001, Proc Instn. Mech. Engrs. Part J, 215, pp. 125-138. 